\title{
TEM of Nanostructured Organic and Hybrid Materials for Photovoltaic and Battery Applications
}

\author{
Jihua Chen \\ Center for Nanophase Materials Sciences, Oak Ridge National Laboratory, Oak Ridge, TN
}

Soft materials including nanostructured organic and hybrid materials are of intense interest and paramount importance in the context of emerging energy technologies including cutting-edge energy storage and energy conversion devices. For decades, polymeric, organic, and relevant hybrid material systems are well known for their poor imaging contrast caused by their low atomic numbers and high electron beam sensitivity resultant from the nature of their weak binding forces. In order to overcome those two obstacles and achieve high-contrast and high-resolution direct imaging of nanostructured organic and hybrid materials in TEM, a combination of low-electron-dose conditions and energy filtering techniques is pursued, which can be widely applicable to organic crystals, polymers, architecturally controlled copolymers, biological macromolecules, composites, and relevant organic-inorganic hybrid systems.

In this work, there are three focused areas related to energy research. They are organic thin film transistors, organic bulk heterojunction based solar cells, and lithium-conducting solid polymer electrolytes. TEM techniques such as low-dose electron diffraction, low eV plasmon mapping, elemental mapping with three-window method are used to correlate device performance, process or crystallization conditions, and nanostructure evolution. In addition, we also discuss about our developments and capabilities in low-dose lattice imaging, scanning helium ion microscopy, and cryogenic energy-filtered TEM.

An example of our work on energy filtered TEM of cutting-edge organic photovoltaic (OPV) devices is shown in Figure 1. A solution-processed, low band gap polymer/fullerene [1] based, high performance OPV active layer $(\sim 100 \mathrm{~nm}$ in thickness) was floated on deionized water followed by subsequent harvesting with a copper grid. A Zeiss Libra 120 TEM with Omega filter was used to examine the bulk heterojunction blend at $120 \mathrm{kV}$ with an emission current as low as $2 \mu \mathrm{A}$ along with other low dose conditions. The sample was monitored closely during elemental mapping using the conventional "three window" method.[2] A typical energy width of $15 \mathrm{eV}$ was used for sulfur mapping and a width of $30 \mathrm{eV}$ was chosen for carbon mapping. Low eV plasmon imaging ( $19 \pm 4 \mathrm{eV}$ for donor and $30 \pm 4 \mathrm{eV}$ for acceptor, data not shown) shows results that are highly consistent with the above-described elemental mapping, both suggesting a nanoscale phase separation between donor-rich and acceptor-rich domains in these high performance OPV systems.[3] 

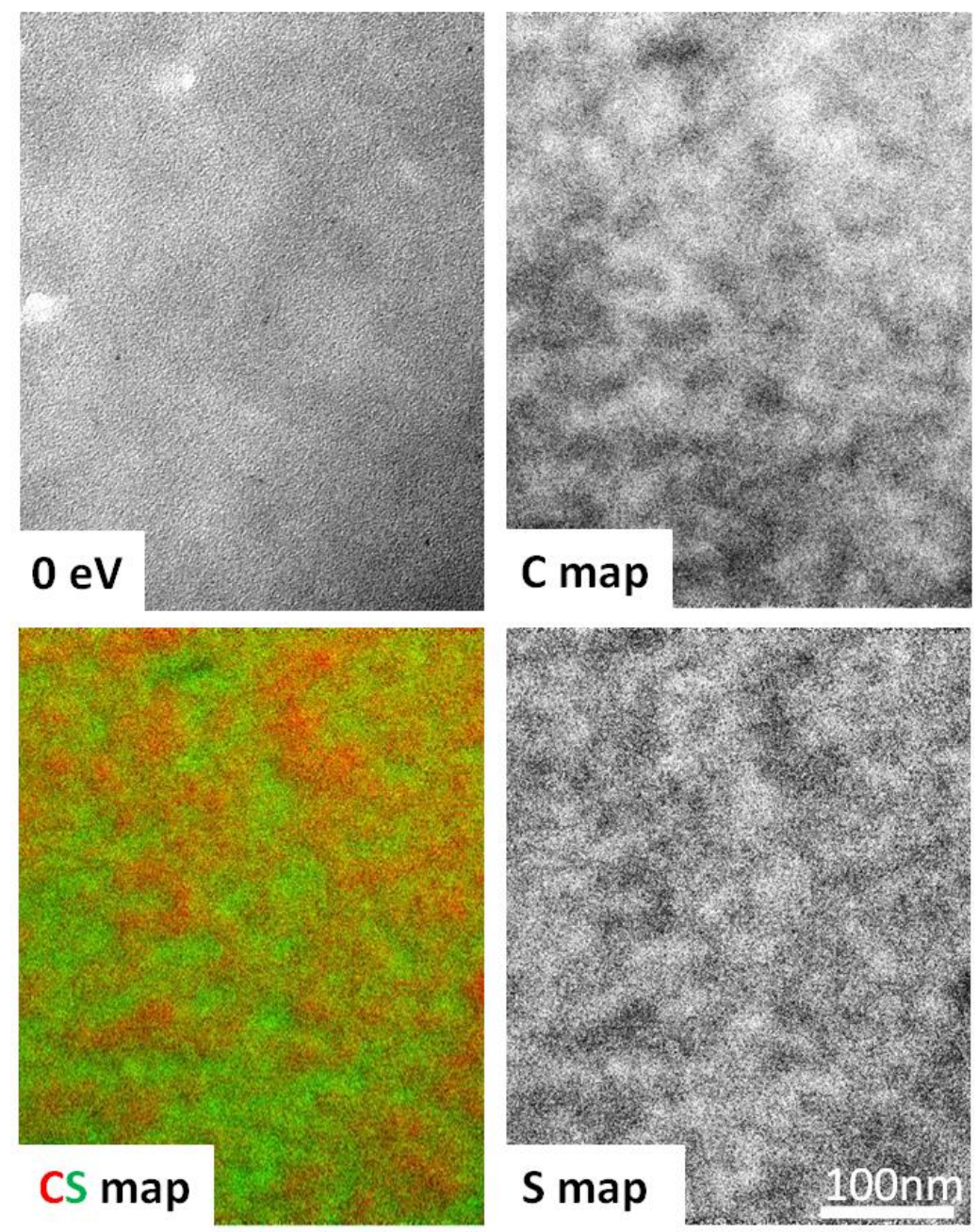

Figure 1. Carbon and Sulfur map generated by the conventional three window method [2] in energy filtered TEM reveals donor and acceptor distribution in a low band-gap polymer $/ \mathrm{PC}_{70} \mathrm{BM}[1]$ based high-performance organic photovoltaic layer. Fullerene-rich domains have higher Carbon density, corresponding to brighter regions in the Carbon map. Polymer-rich domains have higher Sulfur content, which contributes to brighter areas in the Sulfur map. The corresponding thickness map of the same area (data not shown) shows no features at the lengthscale of interest. A $0 \mathrm{eV}$ (elastic) image and composite map are also shown here, with Carbon-rich (or acceptor-rich) areas highlighted in red and Sulfur-rich (or donor-rich) in green. All images are taken from the same sample area, and share the same scale bar at the bottom right.

[1] P Adhikary, et al. Nanoscale 5 (2013), 10007-10013.

[2] RF Egerton, Electron Energy-Loss Spectroscopy in the Electron Microscope, $3^{\text {rd }}$ ed., (Springer, 2011).

[3] This research was conducted at the Center for Nanophase Materials Sciences, which is sponsored at Oak Ridge National Laboratory by the Scientific User Facilities Division, Office of Basic Energy Sciences, U.S. Department of Energy. JC wishes to thank P. Adhikary and Prof. Q. Qiao (South Dakota State University) for providing some of the OPV samples. 